\title{
Assistência de enfermagem no diagnóstico de gastrosquise: uma revisão integrativa
}

\section{de literatura}

\author{
Nursing care in the diagnosis of gastroschisis: an integrative literature review \\ Atención de enfermería en el diagnóstico de gastrosquisis: revisión integrativa de la literatura
}

Recebido: 23/11/2021 | Revisado: 28/11/2021 | Aceito: 29/11/2021 | Publicado: 03/12/2021

\author{
Gicilene Meneses Pimentel \\ ORCID: https://orcid.org/0000-0002-0644-7992 \\ Centro Universitário da Amazônia, Brasil \\ E-mail: gicy.ms2@gmail.com \\ Jamilli Teixeira Nascimento \\ ORCID: https://orcid.org/0000-0003-2229-361X \\ Centro Universitário da Amazônia, Brasil \\ E-mail: jamillynascimento34@gmail.com \\ Adria Maia Leitão \\ ORCID: https://orcid.org/0000-0002-4173-1374 \\ Centro Universitário da Amazônia, Brasil \\ E-mail: adria-maia@hotmail.com
}

\begin{abstract}
Resumo
Objetivo: Apresentar uma revisão de literatura atual acerca dos métodos de abordagem da assistência de enfermagem no diagnóstico de gastrosquise, focando nos cuidados de enfermagem à gestante e os cuidados pré, trans e pós operatório, abordando os principais diagnósticos de enfermagem a serem utilizados no período perioperatório no binômio mãe-bebê. Metodologia: Trata-se de uma revisão integrativa descritiva com abordagem qualitativa, através de análise de artigos relacionados a assistência a gestante com diagnóstico de gastrosquise fetal associado a Assistência de Enfermagem ao neonato no centro cirúrgico, compreendidos entre 25 artigos, sendo selecionados 10 artigos entre 20172020 para composição do quadro e foram utilizados os descritores: Gastrosquise, Assistência de Enfermagem, Centro Cirúrgico e Recém-nascido. Resultados: Há escassez e deficiência na atenção básica quanto ao diagnóstico precoce da Gastrosquise, falta de suporte psicossocial adequado à mãe e familiares, e este em sua integralidade no período gestacional e perioperatório. Entre as principais funções do enfermeiro no perioperatório destaca-se: autorização dos pais para o procedimento cirúrgico, monitorização dos sinais vitais, check-list cirúrgico, manejo do RN em berço aquecido, organizar material para possível reanimação; prestar informações e esclarecer dúvidas aos pais, é importante enfatizar que todo procedimento realizado deve ser registrado em prontuário. Conclusão: Necessita-se desenvolver um modo assistencial específico como protocolo de assistência de enfermagem que envolva condutas e cuidados desde o perioperatório até a possível alta hospitalar; estudos mais aprofundados devem ser realizados com o propósito de qualificar e capacitar a equipe multiprofissional para assegurar assistência livre de erros evitáveis.
\end{abstract}

Palavras-chave: Gastrosquise; Assistência de enfermagem; Centro cirúrgico; Recém-nascido.

\begin{abstract}
Objective: To present a review of current literature on methods of approaching nursing care in the diagnosis of gastroschisis, focusing on nursing care for pregnant women and pre, trans and postoperative care, addressing the main nursing diagnoses to be used in the period perioperative period in the mother-infant binomial. Methodology: This is an integrative descriptive review with a qualitative approach, through the analysis of articles related to care for pregnant women diagnosed with fetal gastroschisis associated with Nursing Care to the neonate in the operating room, comprising 25 articles, with 10 articles being selected among 2017-2020 to compose the table and the descriptors were used: Gastroschisis, Nursing Care, Surgical Center and Newborn. Results: There is a shortage and deficiency in primary care regarding the early diagnosis of gastroschisis, lack of adequate psychosocial support for the mother and family, and this in its entirety in the gestational and perioperative period. Among the main functions of nurses in the perioperative period, the following stand out: parental authorization for the surgical procedure, monitoring of vital signs, surgical checklist, handling the NB in a heated crib, organizing material for possible resuscitation; provide information and clarify doubts to parents, it is important to emphasize that every procedure performed must be registered in the medical record. Conclusion: It is necessary to develop a specific care mode as a nursing care protocol that involves conducts and care from the perioperative period to possible hospital discharge; more in-depth studies should be carried out with the purpose of qualifying and training the multidisciplinary team to ensure assistance free from avoidable errors.
\end{abstract}

Keywords: Gastroschisis; Nursing assistance; Surgery center; Newborn. 


\begin{abstract}
Resumen
Objetivo: Presentar una revisión de la literatura actual sobre métodos de abordaje del cuidado de enfermería en el diagnóstico de gastrosquisis, con foco en el cuidado de enfermería a la gestante y cuidados pre, trans y posoperatorios, abordando los principales diagnósticos de enfermería a ser utilizados en el período perioperatorio en el binomio madrehijo. Metodología: Se trata de una revisión descriptiva integradora con abordaje cualitativo, mediante el análisis de artículos relacionados con la atención a la gestante diagnosticada de gastrosquisis fetal asociada a la Atención de Enfermería al neonato en quirófano, compuesta por 25 artículos, siendo seleccionados 10 artículos entre 2017-2020 para componer la tabla y se utilizaron los descriptores: Gastrosquisis, Atención de Enfermería, Centro Quirúrgico y Recién Nacido. Resultados: Existe escasez y deficiencia en la atención primaria en cuanto al diagnóstico precoz de gastrosquisis, falta de apoyo psicosocial adecuado para la madre y la familia, y esto en su totalidad en el período gestacional y perioperatorio. Entre las principales funciones del enfermero en el perioperatorio se destacan: autorización de los padres para el procedimiento quirúrgico, seguimiento de constantes vitales, checklist quirúrgico, manipulación del RN en cuna climatizada, organización de material para posible reanimación; Brindar información y aclarar dudas a los padres, es importante enfatizar que todo procedimiento realizado debe quedar registrado en la historia clínica. Conclusión: Es necesario desarrollar un modo de cuidado específico como protocolo de cuidados de enfermería que involucre conductas y cuidados desde el período perioperatorio hasta el posible alta hospitalaria; Se deben realizar estudios más profundos con el fin de capacitar y capacitar al equipo multidisciplinar para asegurar una asistencia libre de errores evitables.
\end{abstract}

Palabras clave: Gastrosquisis; Asistencia de enfermería; Centro cirúrgico; Recién nacido.

\title{
1. Introdução
}

De acordo com (Junior et al, 2019), a Gastrosquise é definida como uma malformação congênita da parede abdominal, localizado à direita do cordão umbilical, resultando na extrusão dos órgãos abdominais principalmente no intestino e estômago, geralmente essa malformação da parede abdominal acontece na $4^{\circ}$ à $10^{\circ}$ semana da gestação.

Conforme (Souza et al, 2017), a suposição mais aceitavel para definição da etiologia dessa malformação é o possível incidente causado por isquemia do ventre fetal durante a formação que acontece entre $5^{\circ}$ e a $8^{\circ}$ semana da embriogênese onde ocorre a trajetória nutricional, com o decrescimento da veia umbilical direita a artéria onfalomesentérica direito.

O rompimento embrionário de um desses vasos pode ocasionar isquemia. O diagnóstico precoce através das avaliações e exames no pré- natal faz um grande diferencial na taxa de sobre vida do neonato, possibilitando assim a programação ideal para a grávida e o seu bebê, melhorando os resultados a médio e longo prazo. O planejamento do parto e o nascimento somam-se consideravelmente através das condições assistências do RN. O elemento principal para que soluções sejam tomadas é a informação, que é obtida através de dados informados pela gestante no acompanhamento do pré-natal (Togneri, 2020).

Os estudos mostram que nos últimos anos as taxas de óbitos intrauterino é de 4,48\% nos fetos com diagnóstico de Gastrosquise, essa taxa é muita elevada quando chegamos a comparar com outras malformações, porém a taxa de sobrevivência é de $87,1 \%$ dos registros de nascimento com esse diagnóstico, pois a taxa de sobrevivência da população diminuiria para $80,4 \%$, uma grande diferença entres os países devido a alta renda, que refere-se na taxa de sobrevivência, sendo maior que $90 \%$ (Junior et al, 2019).

De acordo com (Barreiros et al, 2019), aponta que segundo os dados obtidos na América do Sul de 10.000 nascidos 2,9 nascem com a diagnóstico dessa má formação congênita, os fatores que são mais indicados relacionados é a idade Materna, condições sociais, uso de drogas ou álcool e tabagismo. Com o impacto do aumento do número de casos de malformações congênitas, houve uma diminuição de mortalidade infantil por proporcionar novas estratégias de qualidade, com isso o Recém Nascido (RN) é cuidado dentro das melhores condições possíveis e a preparação para o parto e nascimento contribui significativamente na melhoria das condições assistênciais.

As vantagens do diagnóstico precose no Pré-Natal, incluem uma assistência especializada, com preparo e apoio para o nascimento, o prognóstico de vida dos neonatos com gastrosquise são mais elevados, são estabelecidas medidas protetivas e assistênciais antecipada, contribuindo para diminuição nas taxas de mobimortalidade (Almeida et al, 2019). 
Para Oliveira et al, (2017) é recomendado que o tratamento se possível, seja realizado o mais precocemente, com indicação para realização após o parto evitando assim a evaporação das vísceras herniadas que possivelmente podem causar sintomas como hipotermia e infecções neonatais.

Andrade et al, (2017), diz que o RN deve ser submetido ao tratamento cirúrgico, no mínimo com até seis horas de vida, o ideal após o parto é ser encaminhado para uma Unidade de Terapia Intensiva (UTI), sendo necessário que o parto seja realizado em um Hospital de Alta Complexidade, havendo UTI Neonatal e uma equipe multidisciplinar treinada especificamente neste tratamento.

De acordo com (Filho et al, 2018) a gastrosquise pode estar associada a outras malformações e patologias como atresia intestinal e má- rotação intestinal, sendo a ultima diagnósticada em 30- 60\% dos casos.

O aleitamento materno é muito importante para o recém-nascido que nasce com anomalias congênitas e que são submetidos a procedimentos cirúrgicos. Devido ao tamanho desproporcional das vísceras do intestino o procedimento cirúrgico primário se dificulta, sendo realizado de forma progressiva e em vários tempos cirúrgicos (Moreira et al, 2020).

Quando exposto a solicitações cirúrgicas, consequentemente mãe e filho são separados precocemente, esse fator promove dificuldades no aleitamento materno, assim favorecendo o desmame precoce, por isso é importante a rede de apoio para a puerpera, preparando-a para introdução de fórmula lactea, explicando sempre a importância de estimular a criança a sucção no peito. Manter o aconselhamento com comunicação simples e objetiva é primordial (Parola, 2019).

Embora haja inúmeras patologias de malformações congênitas incompatíveis com a vida é necessário o manejo clínico e o cuidado adequados quando se trata da gastrosquise, contudo, assim, será apresentado melhores resultados na sobrevida dos neonatos. As principais condutas nesses casos é a realização do monitoramento para a detecção precoce de sinais e sintomas que indiquem hipotermia, hipotensão, insuficiência respiratória e choque, o mais rápido possível após o nascimento do RN, desde que tenha condições para uma boa recuperação, logo deve-se encaminhar para o tratamento necessário (Barreiros, et al 2019).

A prática do cuidado da enfermagem ao neonato compreende pelo menos três partes: implementação do cuidado, intervenção e interação com outros profissionais de saúde. Se fazendo lembrar da inter-relação desses três componentes com foco na melhoria ou manutenção da saúde dos recém- nascidos e familiares. Margaret Jean Watson, confirmou que o modo de pensar está relacionado ao cuidado e propôs a teoria do cuidado humano (Teoria do relacionamento transpessoal), que considera o cuidado além do tempo, do espaço, do paciente e do profissional, portanto, além dos momentos, também constituem uma forma unificada de interação do elemento, o que é favoravél à recuperação. A equipe de enfermagem desempenha um papel de extrema importância para a manuntenção da vida do RN através do suporte adequado, estabilização do paciente, transporte, mediação de medidas preventivas para que ocorra tudo dentro dos paramêtros corretos (Refrande et al, 2019).

O Recém- nascido deve ser levado em um berço aquecido com calor radiante de modo evitar a perda de calor o mais depressa possível logo após o nascimento. As visceras devem ser introduzidas cuidadosamente em uma bolsa plástico estéril e com solução de soro fisiológico, permitindo a visualização do conteúdo eviscerado. Pode ser observado lesões por isquemias, perfuração, necrose e perda de fluidos. Caso não tenha a bolsa plástica, envolver com gazes embebido com soro fisiológico morno, fazer a introdução da sonda orogástrica para descompressão do estômago, realizar a hidratação venosa imediatamente tendo como base indicada a idade gestacional e o peso. Inicia-se as medicações prescritas pelo médico com antibióticos de amplo aspectro para combater germes da microbiota vaginal materna em caso de parto normal (Albuquerque et al, 2016).

De acordo com o levantamento dos problemas relacionados à demanda de cuidados pode se conhecer e estabelecer os diagnósticos de enfermagem, a partir disso foram listados os principais a serem abordados no diagnóstico de gastrosquise entre eles está o risco de infecção relacionado aos problemas de rompimento da pele e procedimentos invasivos; risco de aspiração relacionada a motilidade gastrointestinal e aumento do resíduo gástrico; risco de desequilíbrio na temperatura corporal relacionada a sedação; risco de perfusão ineficaz relacionado a procedimento cirúrgico ( Campos et al, 2017). 
O Check List de Cirurgia Segura pode ser ajustado conforme as necessidades do serviço, podendo então, ter um específico para os casos de diagnóstico de Gastrosquise, que englobem as necessidades do cuidado no preparo do paciente e da sala cirúrgica, recomenda-se o check list, incluindo três etapas a ser preenchidas: antes da indução anestésica, antes do início do ato cirúrgico e antes da saída do paciente da sala operatória (Caldeira et al, 2017).

Portanto, conclui-se que é essencial que a equipe multiprofissional esteja preparada para receber uma gestante com diagnóstico de gastrosquise e tudo que for necessário para o manejo do neonato deve ser preparado com antecedência e dentro dos padrões satisfatórios para que a cirurgia aconteça sem intecorrências.

Esta pesquisa delimitou como objetivo a abordagem sobre a Assistência de Enfermagem no diagnóstico de gastrosquise, especificando verificar quais os cuidados de enfermagem a gestante em cuidado hospitalar especificamente no Bloco cirúrgico, analisando os cuidados de enfermagem voltados à preparação da sala para recebimento do Recém-nascido, identificando os cuidados no perioperatório de correção da gastrosquise e abordando os principais diagnósticos de enfermagem a serem utilizados.

\section{Materiais e Métodos}

Segundo (Casarin et al, 2020), desde 1980 a revisão integrativa é desenvolvida como metodologia de pesquisa que visa juntar achados de trabalhos teóricos e empíricos, autorizando resultados e buscando entender mais especificamente tal fenômeno respeitando a filiação epistemológica dos trabalhos já concluídos, determinando usar como forma de sistematização e análise para a qualificação permitido solucionar determinada problemática.

Trata-se de uma revisão integrativa descritiva com abordagem qualitativa, através de análise de artigos relacionados a assistência de enfermagem a gestante com diagnóstico de gastrosquise fetal associado a Assistência de Enfermagem ao RN no centro centro cirúrgico. Na estratégia de busca, foram utilizados 25 artigos para referida pesquisa, no período de fevereiro a junho de 2021 e a seleção dos artigos ocorreu através da leitura dos resumos relacionados ao tema em questão, referente a assistência de enfermagem, artigos escritos em português e inglês; periódicos indexados nas bases de dados eletrônicas Biblioteca Virtual em saúde (BVS), Biblioteca Cientifica Eletrônica Online (SciELO) e do Caribe (LILACS); textos com identificação pelo título e resumo; artigos científicos disponíveis gratuitamente e na íntegra nos referidos bancos de dados.

Os critérios de inclusão utilizados foram: os estudos científicos publicados nacionais e internacionais que abordam a temática constante nos bancos de dados. Para o levantamento de dados, foram utilizados as bases de pesquisa Biblioteca Virtual em saúde (BVS), Biblioteca Cientifica Eletrônica Online (SciELO) e do Caribe (LILACS). A faixa anual dos artigos selecionados foram de 2017-2020, tanto na língua inglesa quanto em português sob os seguintes descritores: "Gastroschisis”/ "Gastrosquise”, "Nursing Assistence" / “Assistência de Enfermagem”, "Surgery Center " / "Centro cirúrgico", " newborn " / "Recém-nascido ". Os critérios de exclusão utilizados foram: carta ao leitor, publicações que não se encontravam disponíveis em texto completo, estudo fora do período determinado e que não se encontravam no paramêtro de pesquisa, carta ao editor e/ou que não se encaixavam nos fatores acima, assim os dados foram organizados de forma descritivas.

\section{Resultados e Discussões}

Para elucidar os resultados, foram retratados no Quadro 1 os 10 artigos selecionados. Os dados relacionados são: autores, título do artigo, ano, objetivos e resultados. 
Quadro 1: Síntese dos artigos selecionados.

\begin{tabular}{|c|c|c|c|c|}
\hline $\begin{array}{c}\text { AUTO } \\
\text { RES }\end{array}$ & $\begin{array}{c}\text { TÍTULO DO } \\
\text { ARTIGO }\end{array}$ & ANO & OBJETIVO & RESULTADOS \\
\hline $\begin{array}{c}\text { BARREI } \\
\text { ROS, et } \\
\text { al. }\end{array}$ & $\begin{array}{c}\text { Análise de casos } \\
\text { de gastrosquise } \\
\text { no estado do Rio } \\
\text { de Janeiro }\end{array}$ & 2019 & 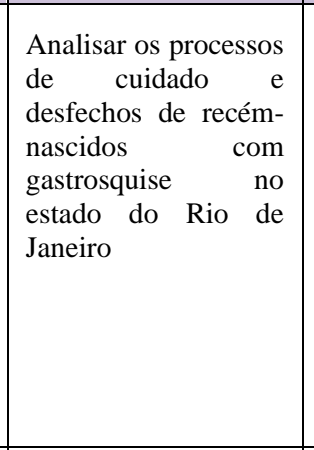 & $\begin{array}{l}\text { Segundo análise no Estado do Rio de Janeiro, concluiu-se que os bebês que } \\
\text { nascem prematuros ou RN abaixo do peso ideal tiveram grandes chances de } \\
\text { vir a óbito, estatisticamente com valor referencial de p: } 0,003 \text { e } 0,006 \\
\text { respectivamente. A prematuridade teve aumento de morte em } 1,6 \text { para } \\
\text { recém- nascidos com gastrosquise. Os RNs com gastrosquise com peso } \\
\text { menor que } 2500 \mathrm{~g} \text { apresentam } 1,8 \text { vezes mais chances de morrer comparados } \\
\text { aos RNs com } 2500 \mathrm{~g} \text { ou mais. Os resultados confirmaram que embora } \\
\text { existam estudos que indicam que as informações e cobertura do SINASC - } \\
\text { Sistema de Informação sobre Nascidos Vivos tenha melhorado } \\
\text { gradativamente, ainda existe falhas importantes da variável de malformações } \\
\text { congênitas, fazendo intensificar a necessidade de profissionais de } \\
\text { enfermagem capacitados para o preenchimento da Declaração de Nascido } \\
\text { Vivo (DNV). }\end{array}$ \\
\hline $\begin{array}{l}\text { MELO, et } \\
\text { al. }\end{array}$ & $\begin{array}{l}\text { Características } \\
\text { epidemiológicas } \\
\text { e clínicas de } \\
\text { gestantes com } \\
\text { fetos com } \\
\text { gastros-quise e } \\
\text { desfechos } \\
\text { perinatais em um } \\
\text { hospital de } \\
\text { referência de } \\
\text { Pernambuco: um } \\
\text { estudo de caso }\end{array}$ & 2019 & $\begin{array}{l}\text { Descrever características } \\
\text { epidemiológicas } \\
\text { clínicas de gestantes com } \\
\text { fetos com gastrosquise e } \\
\text { desfechos perinatais. }\end{array}$ & $\begin{array}{l}\text { O estudo constatou que a maioria das gestantes é parda e solteira, tem peso } \\
\text { pré gestacional adequado e baixa escolaridade com média de idade materna } \\
\text { de } 20,4 \text { anos, mostrou também que quase todas engravidaram pela primeira } \\
\text { vez não havendo registro de aborto anteriormente, sendo o exame realizado } \\
\text { na } 21^{\circ} \text { semana de gestação. Identificou-se que a idade gestacional média para } \\
\text { o parto foi de } 36,7 \text { semanas e a cesariana foi usada na maioria dos casos. } \\
\text { Evidenciado no presente estudo que as doenças obstétricas mais comuns } \\
\text { foram infecção de trato urinário (ITU) e Síndrome Hipertensiva Específica } \\
\text { da Gestação (SHEG). A maioria dos Neonatos precisou de apenas uma } \\
\text { intervenção cirúrgica para cicatrização da gastrosquise e foram classificados } \\
\text { como adequada idade gestacional (AIG), com tempo médio entre o parto e a } \\
\text { cirúrgia de } 1,7 \text { horas e com média de } 18,2 \text { dias de nutrição parenteral. Em } \\
\text { relação à ventilação mecânica um terço dos casos apresentou necessidade e } \\
\text { foram identificadas pelo menos } 12,5 \text { anomalias gastrointestinais associada a } \\
\text { fissura gástrica. O número de óbitos que ocorreu foi de aproximadamente } \\
42 \% \text { dos casos. }\end{array}$ \\
\hline $\begin{array}{c}\text { BARBIER } \\
\text { I, } \\
\text { et al. }\end{array}$ & $\begin{array}{l}\text { Padrão de } \\
\text { crescimento em } \\
\text { fetos com } \\
\text { gastrosquise: } \\
\text { valores de } \\
\text { referência para } \\
\text { paramêtros } \\
\text { ultrassonográfi- } \\
\text { cos e dopplerve- } \\
\text { locimetria das } \\
\text { artérias } \\
\text { umbilicais. }\end{array}$ & 017 & $\begin{array}{l}\text { Avaliar o padrão de } \\
\text { crescimento de fetos } \\
\text { com gastrosquise, } \\
\text { comparando-os aos } \\
\text { fetos normais e } \\
\text { analisar a } \\
\text { dopllerfluxometria } \\
\text { das suas artérias } \\
\text { umbilicais, } \\
\text { correlacionando os } \\
\text { resultados com } \\
\text { dados perinatais. }\end{array}$ & $\begin{array}{l}\text { O presente estudo demonstrou que fetos com gastrosquise apresentam déficit } \\
\text { de crescimento quando comparado aos fetos normais, tendo início no } \\
\text { segundo semestre e é mais significativo no terceiro trimestre da gestação a } \\
\text { restrição do crescimento fetal é a segunda causa de mortalidade perinatal, } \\
\text { está sempre presente em } 5 \text { - } 10 \% \text { de todas as gestações e representa a segunda } \\
\text { causa de mortalidade perinatal responsável por } 30 \% \text { dos natimortos. sabe-se } \\
\text { que os principais fatores de risco maternos relacionados a gastrosquise são a } \\
\text { idade materna precoce, primiparidade, raça branca e baixo IMC. A hipótese } \\
\text { que justifica tais achados é o elevado índice estrogênico no início da gestação } \\
\text { que estão relacionadas a sua gênese. A evisceração ocorre devido a uma } \\
\text { trombose durante o processo natural da involução da veia umbilical direita, } \\
\text { enfraquecendo o local. Os resultados perinatais demonstraram que os fetos } \\
\text { com gastrosquise são constitucionalmente pequenos e não restritos devido a } \\
\text { hipoxia crônica. }\end{array}$ \\
\hline $\begin{array}{l}\text { DIAS, et } \\
\text { al. }\end{array}$ & $\begin{array}{l}\text { Estudo ultrasso- } \\
\text { nográfico pré- } \\
\text { natal na } \\
\text { gastrosquise: que } \\
\text { sinais } \\
\text { influênciam no } \\
\text { desfechos } \\
\text { perinatal }\end{array}$ & 2017 & $\begin{array}{l}\text { Correlacionar } \\
\text { achados das } \\
\text { ultrassonografias } \\
\text { pré- natais na } \\
\text { gastrosquise com a } \\
\text { morbimortali-dade } \\
\text { perinatal, para } \\
\text { avaliar se algum } \\
\text { sinal na } \\
\text { rastreamento no } \\
\text { poderia indicar } \\
\text { maior gravidade. } \\
\text { Caracterizar as } \\
\text { gestantes } \\
\text { diagnosticadas com } \\
\text { gastrosquise } \\
\text { atendidas no } \\
\text { (CIMEFE) e os RNs } \\
\text { intervindos } \\
\text { cirurgicamente no } \\
\text { período neonatal. }\end{array}$ & $\begin{array}{l}\text { Através do estudo pode-se identificar } 33 \text { casos de gastrosquise fetal as } \\
\text { participantes possuíam idade média de } 20,5 \text { anos. Indicou-se que } 50 \% \text { das } \\
\text { gestantes tinham entre } 14 \text { e } 20 \text { anos e outros } 50 \% \text { das gestantes possuíam } \\
\text { entre } 20 \text { e } 33 \text { anos. Das gestantes avaliadas } 24(72,7 \%) \text { eram primigesta } 8 \\
(24,2 \%) \text { secundigesta e } 3,0 \% \text { tercigesta. O diagnóstico da gastrosquise foi } \\
\text { realizado com idade gestacional de } 20,4 \text { semanas com desvio-padrão de } 4,2 \\
\text { semanas. Nos achados ultrassonográficos a dilatação de alças extra- } \\
\text { intestinais foram encontradas em } 16 / 33 \text { ( } 48,5 \%) \text { casos. A presença de } \\
\text { malformações fetais associadas, excluindo as alterações intestinais, foi } \\
\text { descrita em } 7(21,2 \%) \text { casos. Na exteriorização de outros órgãos em conjunto } \\
\text { com as alças intestinais foi descrito o estômago em quatro ( } 12,1 \%) \text { casos. A } \\
\text { bexiga em cinco ( } 15,2 \%) \text { e o fígado em um }(3,0 \%) \text {. A ocorrência de } \\
\text { polidrâmnio e de dilatação da alça intra-abdominal foram relatados em } \\
\text { quatro ( } 12,1 \%) \text { dois exames e oligo-hidrâmnio em três }(9,1 \%) \text {. A distensão } \\
\text { do estomago foi evidenciada em } 2 \text { (6,1\%) dos resultados das } \\
\text { ultrassonografias. Dentro das características fetais foram avaliados } 32 \text { casos, } \\
\text { um dos fatores observados foi o sexo do RNs, sendo } 15 \text { ( } 46,9 \%) \text { do sexo } \\
\text { feminino e } 17 \text { ( } 53,1 \%) \text { masculino; } 22 \text { foram considerados adequados para a } \\
\text { idade gestacional. O índice de apgar no quinto minuto de vida foi maior ou } \\
\text { igual a } 8 \text { em todos os } 32 \text { casos, sendo } 17 \text { recém-nascidos com índice dez, } 12 \\
\text { com índice nove e } 3 \text { RNs com índice oito. Sobre a intervenção cirúrgica } 18 \\
(56,2 \%) \text { foram submetidos ao fechamento por etapas, e em } 14 \text { (e } 43,8 \%) \\
\text { optou-se pelo fechamento primário. Dos recém-nascidos submetidos ao } \\
\text { fechamento primário, } 4 \text { cirurgias foram Exit like. O peso médio variou entre } \\
1475 \mathrm{~g} \text { e } 3480 \mathrm{~g} \text { metade dos mesmos estava abaixo de } 2325 \mathrm{~g} \text {. }\end{array}$ \\
\hline
\end{tabular}




\begin{tabular}{|c|c|c|c|c|}
\hline $\begin{array}{l}\text { ANDRAD } \\
\text { E, et al. }\end{array}$ & $\begin{array}{l}\text { Relato de caso: } \\
\text { gastrosquise e } \\
\text { síndrome do } \\
\text { intestino curto. }\end{array}$ & 2017 & $\begin{array}{l}\text { Buscar fatores que } \\
\text { contribuem para a } \\
\text { morbimortalidade da } \\
\text { doença }\end{array}$ & $\begin{array}{l}\text { O estudo destacou que as principais causas de morbimortalidade na } \\
\text { gastrosquise são diretas ou indiretas ligadas ao dano intestinal iniciado na } \\
\text { vida fetal intrauterina. A doença cursa com déficit nutricional de fluídos e } \\
\text { de eletrólitos, provocando necessidade de reposição agressiva e } \\
\text { monitorização de balanço hídrico. A nutrição parenteral se tornou uma } \\
\text { importante medida em casos de pacientes com menos de } 100 \mathrm{~cm} \text { de intestino } \\
\text { delgado e sem colón, e em pacientes com menos de } 50 \mathrm{~cm} \text { de intestino } \\
\text { delgado e com colón, sendo considerado como tratamento definitivo o } \\
\text { transplante de intestino e nos dias atuais outras técnicas como alongamento } \\
\text { intestinal estão sendo aprimoradas e realizadas. }\end{array}$ \\
\hline $\begin{array}{l}\text { JUNIOR, } \\
\text { et al. }\end{array}$ & $\begin{array}{l}\text { Gastrosquise } \\
\text { fetal: } \\
\text { associação de } \\
\text { trabalho de } \\
\text { parto } \\
\text { espontâneo com } \\
\text { resultado } \\
\text { perinatais } \\
\text { adversos em } \\
\text { fetos termo e } \\
\text { pré-termo. }\end{array}$ & 2019 & $\begin{array}{l}\text { Avaliar a associação } \\
\text { do trabalho de parto } \\
\text { (TP) espontâneo, em } \\
\text { gestações de termo e } \\
\text { pré-termo, com } \\
\text { morbidade } \\
\text { mortalidade } \\
\text { neonatais }\end{array}$ & $\begin{array}{l}\text { O presente estudo demonstrou a relação entre TP e o número de ocorrências } \\
\text { de gastrosquise complexa e de morbidade neonatal. Foi observada nas } \\
\text { gestações de termo a correlação entre trabalho de parto espontâneo e a } \\
\text { necessidade superior de tempo de ventilação mecânica no RN. A ocorrência } \\
\text { de TP espontâneo foi de } 40 \% \text { dos casos e os casos considerados prematuros } \\
\text { a taxa foi de } 57,9 \% \text {. Levando em consideração os subgrupos de partos } \\
\text { prematuros } 63,3 \% \text { sofreram trabalho de parto prematuro (TPP) espontâneo. } \\
\text { Notou-se na análise uma alta taxa de partos cesarianos ( }(91,3 \%) \text {, somente } 18 \\
\text { sendo vaginais. Encontrou-se associação entre TP prematuro e complicações } \\
\text { intestinais pós natais, como a síndrome do intestino curto que sugere } \\
\text { inflamação ou lesão nas alças intestinais do feto no momento do parto. Os } \\
\text { resultados dos estudos perinatais surgiram de um aumento de casos de partos } \\
\text { prematuros nos últimos } 5 \text { anos. Notou-se uma redução considerável de } \\
\text { aproximadamente } 50 \% \text { nos óbitos entre } 2011 \text { e } 2015 \text {, tudo indicou que foi } \\
\text { devido a intensificação da vigilância do bem estar fetal. Não foi identificado } \\
\text { associação entre marcadores ultrassonográficos e a ocorrência de TP } \\
\text { espontâneo. }\end{array}$ \\
\hline $\begin{array}{l}\text { MACHAD } \\
\text { O, et al. }\end{array}$ & $\begin{array}{c}\text { Intervenção } \\
\text { multiprofissional } \\
\text { em paciente com } \\
\text { diagnóstico } \\
\text { de gastrosquise } \\
\text { na unidade de } \\
\text { terapia intensiva } \\
\text { neonatal. }\end{array}$ & 2018 & $\begin{array}{l}\text { Identificar técnicas } \\
\text { utilizadas e o risco de } \\
\text { contaminação. }\end{array}$ & $\begin{array}{l}\text { Segundo estudo a mortalidade e morbimortalidade dessa deformidade são } \\
\text { menores, mas tem se mostrado mais frequentes, com uma estimativa de } \\
\text { 1:200-3:000 por criança nascida. Essa anomalia possui diferentes } \\
\text { apresentações clínicas, enquanto sua etiologia e patogenia permanecem } \\
\text { controversa. O prognóstico depende mais das anomalias cromossômicas ou } \\
\text { anatômicas associadas do que a patologia em si. As principais técnicas } \\
\text { utilizadas atualmente é fechamento primário ou redução gradativa da } \\
\text { deformidade por meio de silos customizados ou pré-formados. O risco de } \\
\text { contaminação diminui consideravelmente com o fechamento primário, } \\
\text { evitando assim sepse, acidose, hipotermia, mas pode ser considerado difícil } \\
\text { dependendo do tamanho da cavidade abdominal, correndo risco de ser } \\
\text { limitado. }\end{array}$ \\
\hline $\begin{array}{c}\text { MOREIR } \\
\text { A, et al }\end{array}$ & $\begin{array}{c}\text { Vivência } \\
\text { materna no } \\
\text { contexto da } \\
\text { amamentação do } \\
\text { recém- nascido } \\
\text { hospitalizado e } \\
\text { submetido a } \\
\text { internação }\end{array}$ & 2020 & $\begin{array}{l}\text { Descrever a vivência } \\
\text { materna no contexto } \\
\text { da amamentação do } \\
\text { filho recém } \\
\text { concebido, } \\
\text { hospitalizado em } \\
\text { Unidade de Terapia } \\
\text { Intensiva Neonatal } \\
\text { (UTIN) e submetido a } \\
\text { intervenção cirúrgica. }\end{array}$ & $\begin{array}{l}\text { O estudo dá ênfase a vivência materna e o vínculo materno que deve ser } \\
\text { estimulado em todos os aspectos nos neonatos, em especial nos casos de } \\
\text { deformidades intestinais ou anormalidades congênitas que necessitam de } \\
\text { cuidados cirúrgicos como a gastrosquise. No estudo, } 8 \text { mães e seus } \\
\text { respectivos RNs foram acompanhados, } 2 \text { bebes ficaram internados } 20 \text { dias, } \\
\text { cinco bebês ficaram entre } 20 \text { e } 24 \text { dias e um bebê ficou } 77 \text { dias. Quando } \\
\text { avaliado o uso de mamadeira sete mães afirmaram não utilizar. Apenas uma } \\
\text { afirmou fazer uso. Em relação ao uso de chupetas seis relataram usar para } \\
\text { fins de alívio de dor. Contudo o fator cirúrgico, instabilidade clínica, dor e a } \\
\text { dependência da aparelhos, deixam sequelas além das cicatrizes nas crianças. } \\
\text { As marcas emocionais geraram insegurança, sentimentos de incapacidade } \\
\text { nas mães e que interferiram diretamente no processo do aleitamento e } \\
\text { nutrição. Da analise das entrevistas surgiram questionamentos considerados } \\
\text { pessoais que estavam relacionados as dificuldades da amamentação, } \\
\text { construção de vínculos na relação mãe- filho e a necessidade de uma rede de } \\
\text { apoio seja familiar ou profissional, o estudo confirmou que todas as mães } \\
\text { que vivenciaram todo o processo de internação do filho, precisavam de } \\
\text { constante apoio e encorajamento. Os profissionais precisam ofertar ajuda } \\
\text { emocional e prática, orientações às puérperas, momentos de educação em } \\
\text { saúde, utilizar as oportunidades para desenvolver açães que promovam o } \\
\text { incentivo ao aleitamento materno mesmo durante a internação e não somente } \\
\text { após a hospitalização. }\end{array}$ \\
\hline $\begin{array}{l}\text { REFRAN } \\
\text { DE, et al. }\end{array}$ & $\begin{array}{l}\text { Vivência do } \\
\text { enfermeiro no } \\
\text { cuidado ao } \\
\text { recém-nascido } \\
\text { com } \\
\text { gastrosquise. }\end{array}$ & 2019 & $\begin{array}{l}\text { Descrever a vivência } \\
\text { do Enfermeiro no } \\
\text { cuidado ao recém- } \\
\text { nascido de alto risco. }\end{array}$ & $\begin{array}{l}\text { Por meio do estudo identificou-se a complexibilidade do manejo dos recém- } \\
\text { nascido de alto risco, sendo realizado através de tecnologias avançadas e um } \\
\text { aprimoramento formado pela teoria e prática compartilhada com os } \\
\text { familiares e os profissionais dentro do universo do neonato, favorecendo uma } \\
\text { abordagem diferenciada e humanizada. Em muitas situações o enfermeiro, } \\
\text { além de realizar um cuidado direcionado ao bebê e a mãe em um papel de } \\
\text { intermediário, aproxima outros familiares para vivenciar esse momento tão } \\
\text { delicado. No entanto, existem alguns desafios que os profissionais da } \\
\text { enfermagem enfrentam como jornada excessiva, trabalho dobrado e exaustão } \\
\text { física que acaba influenciando as vezes negativamente nas tomadas de } \\
\text { decisões e prejudicando no seu exercício. }\end{array}$ \\
\hline
\end{tabular}




\begin{tabular}{|c|c|c|c|c|}
\hline $\begin{array}{l}\text { SANTOS, } \\
\text { et al }\end{array}$ & $\begin{array}{l}\text { Recém- nascido } \\
\text { com } \\
\text { gastrosquise: } \\
\text { história oral de } \\
\text { mães de unidade } \\
\text { de terapia } \\
\text { intensiva } \\
\text { neonatal }\end{array}$ & 2017 & $\begin{array}{ll}\text { Abordar a percepção } \\
\text { de mães cujos recém- } \\
\text { nascidos apresentam } \\
\text { diagnósticos de } \\
\text { gastrosquise } \\
\text { grande porte. }\end{array}$ & $\begin{array}{l}\text { De acordo com a pesquisa e relatos das mães pode-se perceber o quanto é } \\
\text { escasso ainda na atenção básica o cuidado para chegar no diagnóstico } \\
\text { precoce, falta um suporte psicossocial, mais adequado para a mãe e } \\
\text { familiares, que possa permanecer desde o diagnóstico com todo crescimento } \\
\text { sobre a doença até a finalização do tratamento com o procedimento cirúrgico, } \\
\text { além do processo terapêutico conclusivo com alta do RN da UTI. Outro dado } \\
\text { consideravelmente importante foi o reconhecimento da importância da } \\
\text { equipe multiprofissional. A equipe de enfermagem tem papel de desenvolver } \\
\text { um cuidado proximal. No entanto, falta suporte psicossocial para esses } \\
\text { profissionais que se envolvem e presenciam o sofrimento relacionado a mãe } \\
\text { e também ao bebê devido as complicações em alguns casos. Ainda } \\
\text { relacionado ao estudo dentro da temática científica existem poucas } \\
\text { informações de explorações sobre a formas de cuidado ao RN, família e } \\
\text { cuidadores profissionais no contexto gastrosquise. }\end{array}$ \\
\hline
\end{tabular}

Fonte: Autores.

Todos os artigos relatados no Quadro 1 tem ligações diretas e indiretas entre agastrosquise e os cuidados na Assistência de enfermagem, e a partir de uma leitura analítica e minuciosa de cada um, foram destacados pontos de interesse que corroboram para o entendimento e compreensão dos fenômenos e fatos que rodeiam o tema.

Pode-se perceber, escassez na atenção básica quanto ao direcionamento do diagnóstico precoce da Gastrosquise, havendo deficiências significativas de suporte psicossocial adequado à mãe e familiares, e este em sua integralidade no período gestacional e perioperatório, além do processo terapêutico conclusivo com a alta do recém- nascido da UTI. Entre as principais funções da equipe de enfermagem no período perioperatório pode-se destacar: autorização dos pais para o procedimento cirúrgico, monitorização dos sinais vitais, realização de exames físicos, check-list cirúrgico, manejo do RN em berço aquecido e no pósoperatório preparar leito e organizar material para possível reanimação; além de prestar informações e esclarecer dúvidas aos pais, é importante enfatizar que todos os procedimentos devem ser cumpridos e registrado em prontuário.

\section{Considerações Finais}

O presente estudo permitiu esclarecer sobre o manejo da assistência de enfermagem perioperatória em neonatos com a gastrosquise com base em uma assistência e planejamento com qualidade. Foi possível identificar alguns fatores relacionados a prática do cuidado da enfermagem, contudo ainda são pouco realizados nessa temática atualmente, mas foi possível alcançar todas as expectativas. A pesquisa permitiu ressaltar a importância da assistência e cuidados específicos de enfermagem, o diagnóstico precoce no pré-natal que é o grande diferencial relacionado a taxa de mortalidade do RN quanto ao planejamento do nascimento, possibilitando uma preparação para a gestante e o seu bebê.

Sobre a discussão do trabalho, ficou esclarecido a importância do profissional da saúde estar qualificado para prática de uma boa assistência e cuidados específicos, podendo observar diversas características entre as famílias que já convivem com o diagnóstico. Em síntese, os resultados informados através da revisão ajudará no crescimento dos profissionais, de forma a beneficiar a população assistida no centro cirúrgico, procurando primeiramente melhorar a assistência através do que foi colocado em estudo e superar as dificuldades existentes através de todos os cuidados e preparação pautados para criança com diagnóstico de gastrosquise.

A revisão de literatura buscou dar ênfase na importância da humanização dentro da prática assistencial e também nos novos conhecimentos mediante atualizações, existindo a necessidade de buscar compreender mais complexamente o tema, já que é preciso realizar as ações com a equipe multiprofissional para melhor atender o cliente no âmbito cirúrgico,

Em um contexto científico, é importante destacar a necessidade de investimentos em pesquisas que foquem no cuidado do RN com gastrosquise no pré, trans e pós-operatório cujo âmbito seja, voltado para a assitência de enfermagem nesses casos, tal destaque corroboram na falta de publicações científicas relacionadas a temática em destaque, buscando explorar mais formas de desenvolver o modo assistencial específico tanto para a mãe, quanto para o bebê que nasce com gastrosquise. Criar novo 
protocolo para melhorar a assistência de enfermagem, onde envolva condutas e cuidados, desde o período perioperatório até a possível alta hospitalar. Estudos mais aprofundados devem ser realizados com o propósito de qualificar e capacitar a equipe multiprofissional para assegurar uma assistência livre de erros.

\section{Referências}

Albuquerque, R. S. D. Mariani Neto, C., Bersusa, A. A.S. Dias, V. M. \& Silva, M. I. M. d. (2016). Temperatura dos recém-nascidos submetidos ao calor radiante e ao dispositivo Top Maternal ao nascimento. Revista Latino-Americana de Enfermagem, 24. https://www.scielo.br/j/rlae/a/MrsSTcPw3ngkpmNjf9CjkPc/?format=pdf\&lang=pt

Almeida, A.R. (2019). A Atuação Do Serviço Social Junto A Crianças Com Gastrosquise: Algumas Considerações. $16^{\circ}$ Congresso Brasileiro De Assistentes Sociais.Https://Broseguini.Bonino.Com.Br/Ojs/Index.Php/CBAS/Article/View/1229/1202

Andrade, B. C. P. Aguiar, F.P. Lessa, A. B. F. Bhering, A. C., \& Brito Neto, A. C. (2017). Relato De Caso-Gastrosquise e Síndrome do Intestino Curto. Revista UNINGÁ Review, 31(1), 33-34. http://revista.uninga.br/index.php/uningareviews/article/view/2042/1634

Barbieri, M. M. (2017). Padrão De Crescimento em Fetos com Gastrosquise:Valores De Referência Para Parâmetros Ultrassonográficos E Dopplervelocimetria Das Artérias Umbilicais. [Pós-Graduação, Faculdade de Ciências Médicas da Universidade Estadual de Campinas]. UNICAMP. https://bdtd.ibict.br/vufind/Record/CAMP_5a94cf5fed4bfa26e85cd223d00d74f5

Barreiros, C. F. C. Gomes, M. A. S. M. \& Junior, S. C. S. G (2019). Análise Dos Casos De Gastrosquise no Estado do Rio de Janeiro [Doutorado, Instituto Nacional de Saúde da Mulher, da Criança e do Adolescente Fernandes Figueira]. Fundação Oswaldo Cruz. https://www.scielo.br/j/rsp/a/fmVdgJQLJTXXj9JbyZMgPRj/?format=pdf\&lang=pt

Caldeira, L. X. \& Brasileiro, M. E.(2017). Cirurgia Segura: Um Desafio a ser Conquistado. Revista Científica Multidisciplinar Núcleo do Conhecimento. Edição 07. Vol. 01. 44-57. https://www.nucleodoconhecimento.com.br/saude/cirurgia-segura

Campos, D. C. Costa, T. M.Viana,V. P. Melo, A. V. O. G. Catarino, C. F. \& Gazelle, T. G. A (2017). Sistematização Da Assistência De Enfermagem a Um Recém-Nascido Com Gastrosquise: Um Estudo De Caso Clínico. SINADEN, 12, Artigo 12 ${ }^{\mathrm{a}}$ Sinaden. http://www.abeneventos.com.br/12sinaden/anais/resumos/T0378-1.html

Casarin, S. T, Porto, A. R. Gabatz, R. I. B. Bonow, C. A. Ribeiro, J. P. \& Mota, M. S. (2020). Tipos de revisão de literatura: considerações das editoras do Journal of Nursing and Health.. https://periodicos.ufpel.edu.br/ojs2/index.php/enfermagem/article/view/19924

Dias, C. M. (2017). Estudo Ultrassonográfico Pré-Natal na Gastrosquise que Sinais Influenciamno Desfecho Perinatal? [Pós-Graduação- Faculdade de Medicina de São José do Rio Preto]. https://bdtd.famerp.br/bitstream/tede/532/2/CristianedeM.Dias_Dissert.pdf

Filho, A. A. A. C. Coimbra, P. A. Bóscollo-Perez, A. C. Dutra, R. A. \& Alves, K. P. O. A. (2018) Má-rotação intestinal: um diagnóstico a ser considerado no abdome agudo em recém-nascidos.Revista Sociedade Brasileira de Pediatria.. https://cdn.publisher.gn1.link/residenciapediatrica.com.br/pdf/v8n3a08.pdf

Junior, O. G. S. \& Brizot, M. L. (2019). Gastrosquise fetal: associação de trabalho de parto espontâneo com resultados perinatais adversos em fetos de termo e pré-termo.[Universidade de São Paulo-USP] http://www.teses.usp.br/teses/disponiveis/5/5139/tde-14112019-155939/

Machado, C. M. Rocha, A. C. M. Oliveira, B. L. C. Silva, D. E. S. Pereira, F. M., \& Santos,V. T. (2018). Intervenção Multiprofissional em Paciente com Diagnóstico de Gastrosquise na Unidade de Terapia Intensiva Neonatal. [Perspectivas Experimentais e Clínicas, Inovações Biomédicas e Educação em Saúde-PECIBES]. 4(2), 11. https://periodicos.ufms.br/index.php/pecibes/article/view/6904

Melo, M. H. S. T. \& Pinto, N. A. B. (2019). Características Epidemiológicas e Clínicas de Gestantes com fetos com Gastrosquise e desfechos Perinatais em um Hospital de referência de Pernambuco: Um estudo transversal. [Faculdade Pernambucana de Saúde-FPS] http://higia.imip.org.br/bitstream/123456789/349/1/Artigo\%20PIBIC\%202018\%202019_MARIA\%20HELENA\%20SIQUEIRA\%20TAVARES\%20DE\%20 MELO.pdf

Moreira, T. B.Silva, L. R. Silva, M. D. B. Silva, L. J. Mourão, P. P. Moreira, A. P. A. (2020). Vivência materna no contexto da amamentação do recém-nascido hospitalizado e submetido à intervenção cirúrgica. [Escola Anna Nery]. 4. https://www.scielo.br/j/ean/a/jgQPTN8zbCbyPpCkfSVCZhr/?lang=pt\&format=pdf

Oliveira, G. H. Svetliza, J. Vas-Oliani, D. C. M, Júnior, H. L,Oliane,A. H, \& Pedreira, D. A. L.(2017). Nova Abordagem Multidisciplinar para Monitorizar Fetos com Gastrosquise Utilizando o Svetliza Reducibility Index é o procedimento EXIT-like. einstein.vol.(4) 395-402 https://www.scielo.br/j/eins/a/qdmnyHTtXKDJPfBt9tRwqnq/?format=pdf\&lang=pt

Parola, R. A. (2019). Parâmetros Maternos Fetais e Evolução Pós-Natal e Fetos com Gastrosquise. [Pós graduação, Universidade Federal de Minas GeraisUFMG].https://repositorio.ufmg.br/bitstream/1843/32515/1/tese\%20PDFA\%20\%281\%29.pdf

Refrande, S. M. Silva, R. M. C. R. A. Pereira, E. R. Rocha, R. C. N. P. Melo, S. H.S Refrande, N. A. \& dos Santos, R. R. (2019). Vivências do enfermeiro no cuidado a recém-nascido de alto risco: estudo fenomenológico.Revista Brasileira de Enfermagem- REBEN. https://www.scielo.br/j/reben/a/647G3PWkThLyGJPpzLYr9rN/?lang=pt\&format $=$ pdf

Santos, D. S. S. Martins, L. A. (2017). Recém-Nascido Com Gastrosquise: História Oral De Mães Da Unidade De Terapia Intensiva Neonatal. Rev enferm. UFPE on line.Vol (11) https://periodicos.ufpe.br/revistas/revistaenfermagem/article/view/231167/25130

Souza, M. X. Crivaro, E. T. Esteves, T. M. B. Lopes, L. R. S.(2017). Ações Pró-Amamentação para Recém-Nascidos Portadores de Gastrosquise.[ Revista Saúde em Foco] 4, 29-47. http://www4.unifsa.com.br/revista/index.php/saudeemfoco/article/view/1402/1474

Togneri, R. M. Wanderley, H. Y. C. Rodrigues, M. D. C. D. S. Barreto, I. H. A. Queiroz, R. C. M. Pagotti, M. D. Fragoso, A. X. Rebouças, R. G. O., Pereira, A. L. A. D. T. Bortolini, E. R. Errera, F. I. V. (2020). Gastrosquise: incidência e fatores associados. https://downloads.editoracientifica.org/articles/200901559.pdf 